\title{
A
}

Acta HealthMedica

Volume 2, Issue 2, April-June 2017, Pages: 193, DOI: http://dx.doi.org/10.19082/ah193

\section{TRAUMA REGISTRY AT SINA HOSPITAL, TEHRAN, IRAN, 2016: THE FIRST 144 CASES}

Khatereh Naghdi ${ }^{1}$, Sahar Koohi Habibi ${ }^{2}$, Somayeh Bahrami ${ }^{1}$, Zahra Heidari ${ }^{1}$, Mohammad Reza Zafarghandi ${ }^{1}$, Vafa Rahimi-Movaghar ${ }^{1}$, Payman Salamati ${ }^{1 *}$

1: Sina Trauma and Surgery Research Center, Tehran University of Medical Sciences, Tehran, Iran

2: Department of Health Information Management, Tehran University of Medical Sciences, Tehran, Iran

\section{Correspondence:}

Payman Salamati, MD, MPH, Sina Trauma and Surgery Research Center, Tehran University of Medical Sciences, Tehran, Iran. Tel: +98216675002, Fax:+982166757009, E-mail: salamatip@gmail.com

\section{TYPE OF ARTICLE: CONFERENCE ABSTRACT}

\begin{abstract}
Introduction: Injury is one of the main causes of hospitalization, disability and death in Iran. A trauma registry is a modern comprehensive trauma system for injured patients. The present study was designed to evaluate the initial data of traumatic patients admitted at Sina hospital and registered to the National Trauma Registry of Iran (NTRI), Tehran, Iran.

Methods: The inclusion criteria were as follows: patients with acute injury who hospitalized more than 24 hours, injured patients who died after hospital arrival, and patients who were transferred to the ICU of the hospital from another ICU from July through September, 2016. All data were entered in the NTRI's questionnaire.

Results: A total of 144 patients were registered during this time. Of the 144 patients, $125(86.8 \%)$ were male. The mean and standard deviation of patients' age were $37.63 \pm 20.06$ years. The most common mechanism of trauma was traffic accidents in 72 cases (50\%), followed by falling in 32 cases $(22.2 \%)$, penetrating injuries in 26 cases $(18.1 \%)$, blunt injuries in 11 cases $(7.6 \%)$, other transport incidents in 2 cases $(1.4 \%)$ and gunshot in 1 case $(0.7 \%)$. The most common abbreviated injury score level was moderate injury in 81 patients $(56.3 \%)$, followed by minor injury in 37 patients $(27.7 \%)$, serious injury in 17 patients $(11.8 \%)$, severe injury in 6 patients $(4.2 \%)$, critical in 2 patients $(1.4 \%)$, and maximum in 1 patient $(0.7 \%)$. The mean and standard deviation of injury severity score were $5.74 \pm 6.69$.

Conclusion: Young male victims who had traffic accidents with moderate injury accounted for most of our patients. Further studies with larger sample sizes through longer periods of time are recommended.
\end{abstract}

KEYWORDS: Trauma, Injury, Registry.

\footnotetext{
Abstracts of First National Congress of Medical Informatics, Mashhad, Iran, February 2017

(C) 2017 The Authors. This is an open access article under the terms of the Creative Commons Attribution-NonCommercialNoDerivs License, which permits use and distribution in any medium, provided the original work is properly cited, the use is non-commercial and no modifications or adaptations are made. 